\section{$\mathbf{m} / \mathbf{S}$}

méderine/sriences 1994; 10:910

\title{
LES GLYCOPROTÉINES ET LES LECTINES ENDOGĖNES : LE YIN-YANG SUCRÉ
}

es cellules synthétisent une large gamme de structures glucidiques. Quelques-unes de ces structures sont connues depuis de nombreuses années, d'autres viennent seulement d'être décrites, d'autres enfin sont encore inconnues. Pendant très longtemps, les parties glycaniques des glycoprotéines n'intéressaient que les scientifiques spécialisés dans l'étude de la chimie ou de la biochimie des glucides. Au cours des vingt-cinq dernières années, l'importance biologique des glycoprotéines s'est progressivement imposée. En effet, les parties glycaniques des glycoprotéines ne sont pas seulement des éléments passifs (décorations... futiles, ou éléments de protection prévenant l'action des protéases, ou encore éléments de stabilisation de la conformation de la partie protéique), mais elles ont un rôle actif et spécifique en tant que signaux de reconnaissance. Des glycanes complexes, éventuellement substitués par des résidus sulfates ou phosphates, sont reconnus par des récepteurs appelés "lectines". Les lectines sont impliquées dans la reconnaissance entre leucocytes et cellules endothéliales (sélectines), dans les phénomènes infectieux (lectines virales), dans le routage intracellulaire des enzymes lysosomiales (récepteurs spécifiques des oligomannosides phosphorylés), et dans le trafic nucléocytoplasmique et la machinerie moléculaire du noyau (lectines nucléaires). L'importance des glycanes dans ces divers phénomènes, une fois reconnue, ouvre la voie à de nouvelles thérapies met- tant en jeu spécifiquement des oses, des osides, des oligosides et des glycoconjugués.

\section{Sélectines}

Les lectines des leucocytes et des cellules endothéliales jouent un rôle important de reconnaissance dans les phénomènes biologiques fondamentaux, tels que l'arrêt dans les capillaires des lymphocytes, des monocytes et autres leucocytes circulants [1]. Dans les souris transgéniques dont le gène de la sélectine P est invalidé par recombinaison homologue, le comportement des leucocytes dans les capillaires sanguins est altéré : l'extravasion des polynucléaires neutrophiles et leur recrutement au point d'inflammation sont nettement ralentis [2]. I.es structures glycaniques reconnues par les lectines de cellules endothéliales (sélectine E et sélectine P) ont été identifiées comme des oligosaccharides de type I ewis A et/ou Lewis X, contenant du galactose, de l'acide $\mathrm{N}$-acétyl neuraminique et du fucose. Ces oligosaccharides se sont avéré être des protecteurs efficaces contre les phénomènes inflammatoires et des lésions pulmonaires [3]. Ce type de structure glycanique est présent sur divers types cellulaires, en particulier sur certains leucocytes et certaines cellules cancéreuses [4]. Les oliogosaccharides pourraient, en empêchant l'adhérence des cellules tumorales à un endothélium, prévenir la formation de foyers métastatiques. La sélectine L, exprimée à la surface des leucocytes, reconnaît des glycanes sulfatés. Divers ligands natu- 
rels de cette sélectine ont été caractérisés à la surface des cellules endothéliales, le GlyCAM-1 par exemple [5], ou encore le CD34 [6]. Les glycoprotéines possédant des glycanes sulfatés ne constituent pas une exception, même si elles n'avaient pas, jusqu'à un passé récent, retenu l'attention. Par exemple, nombre de glycoprotéines du tissu nerveux contiennent des groupements sulfates. C'est le cas des molécules d'adhérence N-CAM, Po et de la glycoprotéine associée à la myéline, MAG. Un autre exemple est celui de la clusterine, glycoprotéine sulfatée présente dans divers tissus, fortement exprimée dans les phénomènes de neurodégénérescence et dans diverses maladies rénales [7].

\section{Glycoconjugués et lectines des agents infectieux}

Les bactéries sont entourées de polysaccharides qui sont des ligands, soit de lectines solubles, présentes dans les fluides biologiques et membres de la famille des opsonines, soit de lectines membranaires de macrophages, de polynucléaires, de cellules endothéliales, etc. Certaines souches bactériennes expriment des lectines qui permettent à la bactérie d'adhérer aux cellules animales possédant les structures glycaniques ad hoc. Ces processus de reconnaissance ne sont pas limités aux bactéries, puisqu'ils interviennent aussi entre parasites (plasmodium, leishmanies, etc.) et cellules animales, ainsi qu'entre virus et cellules animales. Les lectines du virus de la grippe reconnaissent des glycoprotéines présentant des glycanes liés à l'asparagine et terminés par de l'acide $N$-acétylneuraminique. La lectine du parvovirus B 19 reconnaît un glycane connu sous le nom d'antigène $P$. Ce virus se réplique dans les progéniteurs de la lignée érythroïde qui présentent ce glycane à leur surface. Le parvovirus pourrait, de ce fait, être utilisé pour transférer sélectivement un gène dans les précurseurs de la lignée érythroïde [8]. Le virus de l'immunodéficience acquise VIH se fixe aux cellules $T_{4}$ grâce à la glycoprotéine d'enveloppe gp120 qui intera- git avec la glycoprotéine CD4 cellulaire. Cependant, le virus reconnaît aussi des structures glucidiques de la cellule cible, en particulier le galactosyl céramide [9]. En outre, la glycoprotéine d'enveloppe est très richement glycosylée [10]. Certains de ces glycanes sont reconnus par des lectines: lectines membranaires des macrophages, par exemple, qui favorisent l'endocytose par une voie indépendante du CD4, ou lectine soluble du sérum spécifique du mannose (MBP), qui est une opsonine et qui, une fois fixée, active le complément [11].

\section{Routage intracellulaire des enzymes lysosomiales}

Les enzymes présentes dans les lysosomes sont des glycoprotéines. Au moins un de leurs glycanes lié à une asparagine est du type oligomannose, et au moins un des mannoses est phosphorylé sur l'hydroxyle primaire porté par le carbone 6 . C'est ce glycane phosphorylé qui est reconnu par le récepteur membranaire chargé du routage de ces enzymes de l'appareil de Golgi aux lysosomes. L'absence de phosphorylation se traduit par un déficit fonctionnel majeur des lysosomes, comme dans le cas de la mucolipidose II (maladie à inclusion intracellulaire, I-cell disease). Il y a deux récepteurs spécifiques des oligomannosides phosphorylés dont les principales propriétés ont fait récemment l'objet d'une mise au point [12]. Le récepteur de forte masse moléculaire ( $\mathrm{Mr} 300000)$ connu aussi comme le récepteur de l'un des analogues de l'insuline, l'IGF II - induit, en outre, l'endocytose et le transfert vers les lysosomes des glycoprotéines phosphorylées présentes à l'extérieur des cellules. Diverses maladies lysosomiales sont dues non pas à un défaut de phosphorylation des glycanes oligomannosidiques des enzymes lysosomiales, mais à une mutation du gène codant pour cette enzyme. Par exemple, la mucopolysaccharidose de type I (MPSI, ou maladie de Hurler) est due à un déficit d'activité de l' $\alpha$-L idurodinase. Cette enzyme est indispensable à la dégradation de divers mucopolysaccharides acides, l'héparine en particulier. Chez les individus atteints de cette maladie, le gène est muté $(\mathrm{m} / \mathrm{s}$ $n^{\circ} 11$, vol. 9, p. 1289). Pour ce type de maladies lysosomiales, l'apport d'une enzyme active, portant un glycane oligomannosidique phosphorylé peut être une thérapie efficace. L'enzyme peut être produite $e x$ vivo par des cellules en culture ou in vivo par des cellules implantées [13]. Le récepteur de faible masse moléculaire ( $\mathrm{Mr} 46000)$ ne participe pas à ce mécanisme d'endocytose. Cependant, il est indispensable pour le routage efficace des enzymes vers les lysosomes. En effet, chez des souris transgéniques, dont le gène de cette protéine a été invalidé par recombinaison homologue, une partie importante des enzymes lysosomiales est sécrétée dans le milieu extérieur [14, $15]$.

\section{Glycoprotéines et lectines cytosoliques et/ou nucléaires}

Certaines protéines solubles (non membranaires) du cytosol et/ou du noyau sont glycosylées. Jusqu'à présent, un seul type de glycosylation de ces protéines a été démontré de façon convaincante: il s'agit de la substitution de certains résidus sérine (ou thréonine) par un résidu $\beta$-N-acétyl-glucosamine [16]. Ce type de glycoprotéine a pu être caractérisé sans ambiguïté grâce à la mise au point d'une technique très sensible et très spécifique : la substitution enzymatique de la $N$-acétylglucosamine par un galactose radioactif, suivie d'une ßélimination (coupure de la liaison entre un glycane et un $\beta$-hydroxy aminoacide en milieu alcalin). D'autres formes de glycosylation sont peut-être présentes, mais il faudrait disposer d'une technique appropriée pour les mettre en évidence.

Quelles sont les glycoprotéines cytosoliques ou nucléaires? Diverses classes de glycoprotéines ont été caractérisées [17]: les facteurs nucléaires Spl, AP1, etc. [18], et des enzymes, dont l'ARN polymérase II [19]. La 
partie C-terminale de cette enzyme est en effet fortement glycosylée et phosphorylée lorsqu'elle est dans le complexe d'initiation; elle est, en revanche, totalement déglycosylée et phosphorylée lorsqu'elle est dans le complexe d'élongation. $\mathrm{La} O$ glycosylation intracytosolique et/ou nucléaire des protéines solubles apparaît ainsi, à côté de la phosphorylation, comme un élément fondamental de la régulation de la machinerie cellulaire.

De façon complémentaire, des lectines nucléaires et/ou cytosoliques ont été caractérisées. L'une d'entre elles, la CBP35, qui est spécifique des galactosides, est associée aux hnRNP [20] ; ces complexes passent du noyau dans le cytosol. A l'inverse, lorsque l'on introduit des glycoprotéines dans le cytosol, elles migrent dans le noyau grâce à un mécanisme qui nécessite de l'énergie et qui dépend de la nature de l'ose exposé, mais qui est indépendant du chemin utilisé par les peptides de localisation nucléaire ([21] et résultats non publiés).

\section{Oligosaccharides et analogues de glycoconjugués comme agents thérapeutiques}

Sur la base des résultats accumulés au cours de ces dernières années, diverses molécules contenant des oses, des glycanes ou certains de leurs dérivés sont potentiellement des agents thérapeutiques. Nous avons vu plus haut que des oligosaccharides reconnus sélectivement par la sélectine $P$ sont actifs comme agents protecteurs dans des phénomènes inflammatoires. Un analogue de l'acide $N$-acétylneuraminique [22] inhibe efficacement l'invasion des cellules par le virus de la grippe $\left(\mathrm{m} / \mathrm{s} n^{\circ}\right.$ 11, vol. 9, p. 1270). Les oses et les oligosaccharides spécifiques reconnus par des lectines exprimées sélectivement à la surface de certaines cellules peuvent être utilisés efficacement pour favoriser le transfert de gènes dans ces cellules en utilisant des polylysines glycosylées [23].

Les parties glycaniques des glycoprotéines conditionnent leur fonction et $\mathrm{m} / \mathrm{s} n^{\circ} 1$ vol. 10, janvier 94 leur destinée. La structure des glycanes dépend à la fois de la protéine, et de la cellule qui l'exprime. Cela a deux conséquences majeures: (1) une glycoprotéine peut exister sous la forme de plusieurs variants (glycoformes); (2) l'activité d'une protéine recombinante peut être fortement compromise si elle n'est pas exprimée dans la cellule ad hoc. Les animaux transgéniques obtenus par invalidation d'un gène de lectine, d'une glycosidase ou d'une glycosyltransférase devraient faire progresser très rapidement la connaissance du rôle des lectines et des glycanes qui sont, rappelons-le, synthétisés à l'aide des diverses transférases. Enfin, la connaissance précise de la structure des glycanes complexes reconnus spécifiquement par les lectines permettra le développement rationnel de nouveaux médicaments et favorisera l'introduction sélective de gènes dans les cellules exprimant, soit un glycane particulier, soit une lectine particulière. La glycobiologie entre ainsi dans l'ère de la transgénèse et de la thérapie génique

\section{RÉFÉRENCES}

1. Durand (;. Les sélectines et leur rôle dans l'interaction des leucocytes avec les cellules endothéliales, médecine/sciences $1992 ; 8: 1051-6$

2. Mulligan MS, Paulson JC, De Frees S, et al. Protective effects of oligosaccharides in P selectin dependent lung injury. Nature $1993 ; 364: 149-51$

3. Mayadas TN, Johnson RC, Rayburn H, Hynes RO, Wagner DD. Leukocyte rolling and extravasation are severely compromised in P selectin deficient mice. Cell 1993; 74: 541-54.

4. Takada A. Ohmori K, Yoneda T, et al. Contribution of carbohydrate antigens sialyl Lewis $\mathrm{A}$ and sialyl Lewis $\mathrm{X}$ to adhesion of human cancer cells to vascular endothelium. Cancer Res 1993; 53 : 354-61. 5. Imai Y, Lasky LA, Rosen SD. Sulphation requirement for glyCAM-1, an endothelial ligand for L selectin. Nature 1993; 361: 555-7.

6. Baumhueter S, Singer MS, Henzel W, et al. Binding of L-selectin to the vascular sialomucin (.D34. Srience 1993; $262: 436-8$.
7. Rabenandrasana C,, Ramsohoye P. La clusterine: une nouvelle glycoprotéine protectrice de l'intégrité cellulaire? médecine/sciences 1993 ; 10 : 12-20.

8. Brown KE, Anderson SM, Young NS. Erythrocyte $\mathrm{P}$ antigen : cellular receptor for B19 parvovirus. Science 1993; 262: 114-7. 9. Fantini J, Yahl N. Le galactosylceramide : un nouveau récepteur pour le virus de l'immunodéficience humaine (VIH). médecine/sciences 1993; 9 : 891-900.

10. Fenouillet E. La N-glycosylation du VIH : du modèle expérimental à l'application thérapeutique. médecine/sciences 1993 ; 9: 901-6.

11. Haurum JS, Thiel S, Jones IM, et al. Complement activation upon binding of mannose binding protein to HIV envelop glycoproteins. AIIJS 1993; 7 : 1307-13.

12. Méresse S, Bauer U, Ludwig T, et al. Bases moléculaires du transport vers les lysosomes. médecine/sciences $1993 ; 9$ : 148-56. 13. Moullier $P$, Maréchal V, Danos $O$, Heard JM. Continuous systemic secretion of a lysosomal enzyme by genetically modified mouse skin fibroblasts. Transplantation 1993 ; 56 : 427-32.

14. Ludwig T, Ovitt (.E, Bauer U, et al., Targeted disruption of the mouse cationdependent mannose 6-phosphate receptor results in partial missorting of multiple lysosomal enzymes. $E M B C$ J 1993 ; 12 : 5225-35.

15. Kōster A, Saftig P, Matzner U, el al. Targeted disruption of the $\mathrm{Mr} 46000$ 6 -phosphate receptor gene in mice results in misrouting of lysosomal proteins. EMBO/ 1993 ; 12 : 5219-23.

16. Holt (;D, Hart (; W'. Subcellular distribution of terminal $\mathrm{N}$ acetylglucosamine moieties. Localization of a novel proteinsaccharide linkage (O-linked GlcNAc. / Biol (.hem 1986; 261: 8049-57.

17. Haltiwanger RS, Kelly WG; Roquemore EP, et al. Glycosylation of nuclear and cytoplasmic proteins is ubiquitous and dynamic. Biochem Soc Transactions 1992; 20: 264-9.

18. Jackson SR, Tjian R. O glycosylation of eukaryotic transcription factors: implications for mechanisms of transcriptional regulation. Cell 1988; 55 : 125-33.

19. Kelly WG, Dahms ME, Hart (;W. RNA polymerase II is a glycoprotein. Modification of the $\mathrm{COOH}$-terminal domain by $\mathrm{O}$ GIcNAc. J Biol Chem 1993; 268 : 10416-24. 20. Wang JI, Laing JG, Anderson RL. Lectins in the cell nucleus. Glycobiology 1991; 1 : 24.3-52.

21. Duverger E, Carpentier V, Roche AC, Monsigny M. Sugar dependent nuclear import of glycoconjugates from the cytosol. Exp Cell Res 1993; 207 : 197-201.

22. von ltzsein $M, W u W Y, K o k$ GB, et al. Rational design of potent sialidase-based inhibitors of influenza virus replication. Nature 1993; 363: 418-23.

23. Monsigny $M$, Midoux $P$, Roche AC. Perspectives ex vivo et in vivo, pour la thérapie génique, de la transfection sélective à l'aide de complexes plasmide-polylysine ciblés. médecine/sciences 1993; 9: 441-9. 